\title{
SURFACE STRUCTURE AND ANALYSIS WITH SCANNING PROBE MICROSCOPY AND ELECTRON TUNNELING SPECTROSCOPY
}

\author{
Final Report
}

Julia W. P. Hsu

\section{University of Virginia}

Charlottesville, Virginia 22901

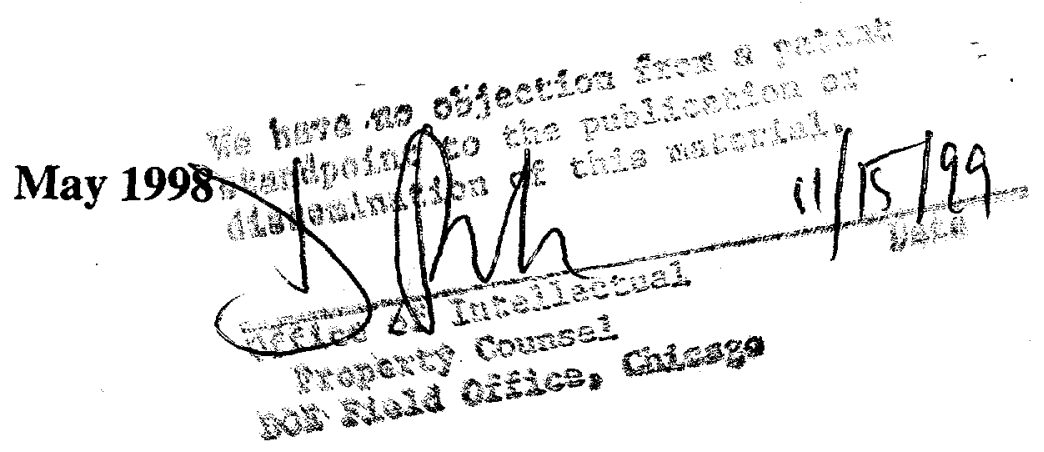

Prepared for

The U.S. DEPARTMENT OF ENERGY

AWARD NO. DE-FG0\$-84ER45072

2 


\section{DISCLAIMER}

This report was prepared as an account of work sponsored by an agency of the United States Government. Neither the United States Government nor any agency thereof, nor any of their employees, make any warranty, express or implied, or assumes any legal liability or responsibility for the accuracy, completeness, or usefulness of any information, apparatus, product, or process disclosed, or represents that its use would not infringe privately owned rights. Reference herein to any specific commercial product, process, or service by trade name, trademark, manufacturer, or otherwise does not necessarily constitute or imply its endorsement, recommendation, or favoring by the United States Government or any agency thereof. The views and opinions of authors expressed herein do not necessarily state or reflect those of the United States Government or any agency thereof. 


\section{DISCLAIMER}

Portions of this document may be illegible in electronic image products. Images are produced from the best available original document. 
This report summarizes the results accomplished during the funding period of this grant (June 1, 1995 to May 31, 1998). The projects are (1) room-temperature atomic force microscopy (AFM) studies of $\mathrm{NbSe}_{3}$ doped with various elements and (2) low-temperature scanning tunneling microscopy (STM) studies of $\mathrm{NbSe}_{3}$. In addition, AFM was used to study the surface morphology and defects of GaAs films grown on $\mathrm{Ge}$ and $\mathrm{Ge} / \mathrm{Si}$ substrates.

There are no unexpended funds at the end of the funding period.

\section{(1) AFM studies of various dopants in $\mathrm{NbSe}_{3}$}

Pure $\mathrm{NbSe}_{3}$ has two CDW transitions with onset temperatures $\mathrm{T}_{\mathrm{H}}=144 \mathrm{~K}$ and $\mathrm{T}_{\mathrm{L}}=59 \mathrm{~K}$. Pure $\mathrm{NbSe}_{33}$ has a monoclinic crystal structure with three identical chain pairs per unit cell. Previous scanning tunneling microscope (STM) results at $4.2 \mathrm{~K}$ indicate that the low-temperature CDW produces a stronger modulation of the electron density on both chain pairs I and II than that caused by the high-temperature CDW on chain pair III. The high-temperature CDW is in phase in adjacent unit cells, while the low-temperature CDW is out of phase with adjacent unit cells. When $\mathrm{NbSe}_{3}$ is doped with different elements, its charge density wave (CDW) properties change. For many elements, that form interstitial dopants in $\mathrm{NbSe}_{3}$, a few percent of impurity results in long range modulations of surface electronic at room temperature, a temperature that is much higher than the onset of CDW. These surface undulations can be observed using AFM. Studies have been done on $\mathrm{NbSe}_{3}$ doped with $\mathrm{Co}, \mathrm{Cr}, \mathrm{Fe}, \mathrm{Gd}, \mathrm{Mn}, \mathrm{Ni}, \mathrm{Pd}$, and $\mathrm{V}$. Ti forms substitutional instead of interstitial dopant in $\mathrm{NbSe}_{3} . \mathrm{NbSe}_{3}$ doped with $\mathrm{Ti}$ does not show any long range surface modulations.

This topic is the core of $\mathrm{PhD}$ dissertations for several of the late $\mathrm{Dr} . \mathrm{R}$. V. Coleman's students. Y. Gong, J. P. Qian, and D. L. Drake have been supported partially by this grant. Since Dr. Coleman's death, Y. Gong and J. P. Qian have completed their PhD dissertations. Their findings on $\mathrm{NbSe}_{3}$ doped with the elements given in the previous paragraph have been published ${ }^{1}$ and reported previously; hence, they will not be repeated here. Most recently, the changes in CDWs in $\mathrm{W}_{\mathrm{x}} \mathrm{NbSe}_{3}$ and $\mathrm{Si}_{\mathrm{x}} \mathrm{NbSe}_{3}$ have been studied by D. L. Drake. He presented the findings in a talk during the 1996 American Physical Society Meeting at St. Louis. Presently, all experimental work 
has been completed. However, due to personal reasons, D. L. Drake has not yet completed writing his thesis. The outline of his thesis is attached to this report. He has completed Chapters 2 and 3; Chapter 4 is mostly done, and Chapter 5 is partially done. We expect that he will be able to defend his dissertation in the next 6 months.

\section{(2) Low-temperature STM studies of $\mathrm{Pd}_{x} \mathrm{NbSe}_{3}$}

This project was the core of J. P. Qian's PhD research. He studied the changes in CDW as a function of Pd concentration at $4.2 \mathrm{~K}$. The scientific results of this study have been reported in the previous progress report and will not be repeated here.

\section{(3) AFM studies of GaAs films on Ge and Ge/Si substrates}

In addition to study $\mathrm{NbSe}_{3}, \mathrm{AFM}$ was used to study the surface morphology of GaAs films grown on $\mathrm{Ge}$ and $\mathrm{Ge} / \mathrm{Si}$ substrates. GaAs/Ge films are technologically important and have been used to fabricate many electronic and optoelectronic devices, such as high-efficiency solar cells, phototransistors, and bipolar transistors. The high quality of GaAs films grown on Ge substrates is crucial for the performance of these devices. Molecular beam epitaxy (MBE) has been shown to make high quality heteroepitaxial films, due to its atomic level control. However, defects can still be generated because the growth of GaAs on Ge is complex. For example, even though the lattice mismatch between $\mathrm{Ge}$ and $\mathrm{GaAs}$ is less than $0.1 \%$, threading dislocations can originate from interfacial As point defects. In addition, anti-phase domains (APDs) can appear because GaAs is polar while the Ge substrate is non-polar. Thus, it is challenging to obtain optimal condition for growing defect-free GaAs films on Ge. Using the AFM, we study how various growth parameters affect the surface morphology and roughness of MBE grown GaAs/Ge films. The results are correlated with defect studies from scanning electron microscopy (SEM) and transmission electron microscopy (TEM) and structural characterizations from $\mathrm{X}$ ray and in situ reflection high energy electron diffraction (RHEED).

Figure 1 shows the AFM topographical images of two $1000 \AA$ thick GaAs films grown on $\mathrm{Ge} / \mathrm{Si}$ substrates, depicting the effect of prelayers, $\mathrm{Ga}$ or As, on the defect formation and surface 
morphology. Figures 1(a) and (c) are for a Ga prelayer sample and Figs. 1(b) and (d) are for a As prelayer sample. The scanning areas shown in Fig. 1(a) and (b) are $(10 \mu \mathrm{m}) \times(10 \mu \mathrm{m})$ and those in Fig. 1(c) and Fig. (d) are $(2.5 \mu \mathrm{m}) \times(2.5 \mu \mathrm{m})$. Figure 1(a) and (b) show that both samples bear the same large scale background undulation patterns with characteristic length scale $\sim 1 \mu \mathrm{m}$. On top of this mild undulation, the Ga prelayer sample shows isolated dark spots with density 5 $\times 10^{8} \mathrm{~cm}^{-2}$. Most of these are threading dislocations generated when $\mathrm{Ge}$ was grown directly on $\mathrm{Si}$ substrates. The As prelayer sample displays a complicated pattern of dark lines (Fig. 1(b)). At higher resolution (Fig. 1(d)), one can see that these dark lines segregate into irregularly shaped closed loops in some regions. We also find regions where the lines are continuous at $\sim 1 \mu \mathrm{m}$ scale. TEM studies have established that these lines are anti-phase boundaries (APBs), which separate growth domains of reversed polarities with respect to the substrate orientation by a $90^{\circ}$ rotation about the [001] axis. The APBs appear dark in the AFM images, which means that their surface morphology is depressed. The root-mean-square roughness $\left(\sigma_{\mathrm{rms}}\right)$ for the $\mathrm{Ga}$ and As prelayer samples are $\sim 25 \AA$ and $\sim 45 \AA$. These AFM results indicate that Ga prelayer samples are superior: smoother with no apparent anti-phase defects. However, on a close examination, we found that not all dark spots in Fig. 1(a) are simple pits (Fig. 1(c)). Some of them are loops similar to APBs on the As prelayer sample (Fig. 1(d)). Based on the similarity, we conclude that there are also APBs on the Ga prelayer sample. As GaAs films grow thicker, the APBs annihilate each other. At the surfaces of $1 \mu \mathrm{m}$ thick films, there are no APBs. However, the As prelayer film is much rougher, $\sigma_{\mathrm{rms}} \sim 115 \AA$, than the Ga prelayer film $\left(\sigma_{\mathrm{rms}} \sim 30 \AA\right){ }^{2}$

We also studied the effect of Ge buffer when growing GaAs films on Ge wafers. Fig. 2 compares the surface topographical images of GaAs samples without a Ge buffer layer (Fig. 2(a)) and with an annealed Ge buffer layer (Fig. 2(b)). Both samples have Ga prelayers and are $1000 \AA$ thick. The scanning areas are $5 \mu \mathrm{m} \times 5 \mu \mathrm{m}$. The sample grown directly on a Ge wafer (Fig. 2(a)) shows many pits and dark loops on its surface. These loops are surface APBs. TEM results also confirms that APBs reach the surface in this sample. On the contrary, the sample grown on an annealed Ge buffer layer (Fig. 1(b)), shows only pits on the surface. The $\sigma_{\text {rms }}$ is $9 \AA$ for the 
sample in Fig. 2(a) and $3.5 \AA$ for the sample in Fig. 2(b). This confirms that APBs make sample surface rougher. The two As prelayered samples of the same thickness and grown under the same conditions as the Ga prelayered samples shown in Fig. 2 do not show big differences on the SFM topographical images. From $\sigma_{\text {rms }}$ numbers above, we see that the samples with an annealed Ge buffer layer are relatively smoother than the samples without a Ge buffer layer even though the difference is not large for these thin films. The difference is significantly larger for $2.5 \mu \mathrm{m}$ thick films. ${ }^{3}$ The pit densities are approximately $10^{9} / \mathrm{cm}^{2}$. After comparing this with the TEM results, we conclude that these pits are threading dislocations punch through the sample surface. The cause of such high density threading dislocations is not related to the Ge buffer layer, but is due to As point defects generated during the low temperature $\left(350{ }^{\circ} \mathrm{C}\right)$ growth of the initial $1000 \AA$ thick GaAs films. Raising the growth temperature to $500{ }^{\circ} \mathrm{C}$ for the first $1000 \AA$ reduces the threading dislocation density below the detection limit of TEM $\left(\leq 10^{7} / \mathrm{cm}^{2}\right)$.

We also studied the effect of annealing Ge buffers at a higher temperature on GaAs surface morphology for $\mathrm{d}_{\mathrm{GaAs}}=1 \mu \mathrm{m}$ samples with either prelayers ( $\mathrm{Ga}$ or As). The sample shown in Fig. 3(a) has an unannealed Ge buffer layer, while then sample shown in Fig. 3(b) has an annealed Ge buffer layer before GaAs deposition. Both samples are $\mathrm{Ga}$ prelayer and the scanning areas are $10 \mu \mathrm{m} \times 10 \mu \mathrm{m}$. Fig. 3(a) is very rough $\left(\sigma_{\mathrm{rms}}=340 \AA\right)$, with facets on the entire surface of the sample. The average lateral sizes of these facets are from 0.6 to $0.8 \mu \mathrm{m}$; the slope varies from $8^{\circ}$ to $30^{\circ}$. The corresponding As prelayer sample shows similar surface features, i.e., a rough surface with facets, but it is not as severe $\left(\sigma_{\mathrm{rms}}=23 \AA\right)$. In addition, there are crevices and elongated mounds on its surface. The Ga prelayer sample with an annealed Ge buffer layer is shown in Fig. 3(b), and the corresponding As prelayered sample has a very similar topography. They are both very smooth with $\sigma_{\mathrm{rms}}=5.5 \AA$. The uniform elongated mounds observed on the sample surfaces are typical GaAs homoepitaxial growth morphology as described in Ref. 4. In 80 $\mu \mathrm{m} \times 80 \mu \mathrm{m}$ AFM images, we do not observe any crosshatch patterns on samples (with either prelayers) with unannealed Ge buffer layers, while we see obvious crosshatch patterns on samples with annealed Ge buffer layers. This result can be explained by the extensive APBs on samples 
with unannealed Ge buffer layers, compared with samples with annealed Ge buffer layers. RHEED patterns of GaAs films grown on annealed Ge buffer layers display a GaAs $2 \times 4$ reconstruction, indicating a single domain GaAs. On the contrary, RHEED patterns of GaAs films on unannealed Ge buffer layers show mixed GaAs $2 \times 4$ and $4 \times 2$ reconstructions, indicating a mixture of GaAs domains, i.e., the presence of APBs. The APBs on samples with unannealed Ge buffer layers blocked the misfit dislocation glide ${ }^{5}$ and destroy the corresponding crosshatch patterns completely. We conclude that the high temperature annealing of the Ge buffer layer greatly reduces the generation of APBs during the GaAs growth.

To study how surface morphology evolves as GaAs grows thicker, we compared $1 \mu \mathrm{m}$ and $2.5 \mu \mathrm{m}$ thick samples. All samples have an annealed Ge buffer layer. Figures 4(a) and (b) show As prelayer samples imaged at $80 \mu \mathrm{m} \times 80 \mu \mathrm{m}$ scale, and Figs. 4(c) and (d) at $5 \mu \mathrm{m} \times 5 \mu \mathrm{m}$ scale. Figs. 4(a) and (c) are for the $1 \mu \mathrm{m}$ thick sample, and Figs. 4(b) and (d) are for the $2.5 \mu \mathrm{m}$ thick sample. As mentioned before, Figs. 4(a) and (b) display crosshatch lines characteristic of low lattice mismatch systems. These samples are thick enough to exceed the critical thickness (about $0.29 \mu \mathrm{m}$ to $0.5 \mu \mathrm{m})^{6}$ so that misfit dislocations are generated. The crosshatch lines are directly related to the presence of these misfit dislocations. The only difference between the $1 \mu \mathrm{m}$ and 2.5 $\mu \mathrm{m}$ thick samples is that the crosshatch lines on the $2.5 \mu \mathrm{m}$ thick samples are denser, shorter, and deeper. The crosshatch line height changes are very small $(\sim \mathrm{nm})$, which makes it difficult to detect them by SEM or optical microscopy. Unlike these techniques, AFM can directly measure them due to its sub- $\AA$ sensitivity. Figures 4 (c) and (d) show $5 \mu \mathrm{m} \times 5 \mu \mathrm{m}$ AFM images of the same samples shown in Figs. 4(a) and (b), respectively. Elongated mounds or growth islands are observed to be uniformly distributed on the surface with long axis along the $\left[{ }^{{ }^{-}} 10\right]$ direction, as determined by referring to the orientation of the crosshatch lines in the large scale AFM images. We find that the orientation of these mounds is independent of the prelayers during the $\mathrm{MBE}$ growth. By performing auto-correlation studies on these samples, we found that the elongated mounds on the $1 \mu \mathrm{m}$ thick samples are $0.8 \mu \mathrm{m} \times 1.1 \mu \mathrm{m}$ (Ga prelayer) and $0.9 \mu \mathrm{m} \times 1.3 \mu \mathrm{m}$ (As prelayer). The elongated mounds on the $2.5 \mu \mathrm{m}$ thick samples are both around $1 \mu \mathrm{m} \times 2 \mu \mathrm{m}$ for 
$\mathrm{Ga}$ and As prelayer samples. The slopes for the mounds on all these samples remain about 0.3 $0.5^{\circ}$. These values are close to the similar mounds observed on homoepitaxial MBE GaAs samples. ${ }^{3}$ The $\sigma_{\mathrm{rms}}$ increases as the $\mathrm{d}_{\mathrm{GaAs}}$ increases. The annealed Ge buffer layers of these thick samples provide a single domain substrate upon which the growth initiates.

' Y. Gong, Q. Xue, D. L. Drake, J. Qian, and R. V. Coleman, Phys. Rev. B 51, 975 (1995); Q. Xue, Y. Gong, D. L. Drake, J. Qian, and R. V. Coleman, Phys. Rev. B 53, 1068 (1996)

${ }^{2}$ Q. Xu, J. W. P. Hsu, E. A. Fitzgerald, J. M. Kuo, Y. H. Xie, and P.J. Silverman, J. Elec. Mater. 25, 1009 (1996)

${ }^{3}$ Q. Xu, J. W. P. Hsu, S. M. Ting, E. A. Fitzgerald, R. M. Sieg, and S. A. Ringel, J. Elec. Mater. (in press)

${ }^{4}$ C. Orme, M. D. Johnson, J. L. Sudijono, K. T. Leung, and B. G. Orr, Appl. Phys. Lett. $64,860(1994)$

${ }^{5}$ S. M. Ting, E. A. Fitzgerald, R. M. Sieg, and S. A. Ringel, J. Elec. Mater. (in press)

${ }^{6}$ P. Franzosi, L. Lazzarini, G. Salviati, M. Scaffardi, and G. Timo, Inst. Phys. Conf. Ser. 117: Section 7; C. Bocchi, B. Bollani, P. Franzosi, L. Laazarini, D. Passoni, and G. Timi, ibid, Section 9 
Impurity Effects in NbSe3: A Transport and Atomic Force Microscope Study by David Lawrence Drake

Abstract

Acknowledgments

Chapter 1: Introduction

Chapter 2: Experimental Techniques and Equipment

2.1 Crystal Growth and Properties

2.2 Crystal Mounting Techniques/Sample Preparation

2.3 Sample Holders and Measurement Techniques

2.4 AFM Design and Techniques

Chapter 3: Theory

3.1 Charge Density Waves

3.2 CDW Fluctuations above $T_{c}$ and Impurities

3.3 Transport

3.4 Atomic Force Microscopy

Chapter 4: Transition Metal Trichalcogenides

4.1 General $\mathrm{MX}_{3}$ Characteristics

$4.2 \mathrm{NbSe}_{3}$

4.3 Impurity-Induced New Structures

Chapter 5: Experimental Results and Discussion

5.1 Transport Results

5.2 AFM Results

Chapter 6: Conclusions 

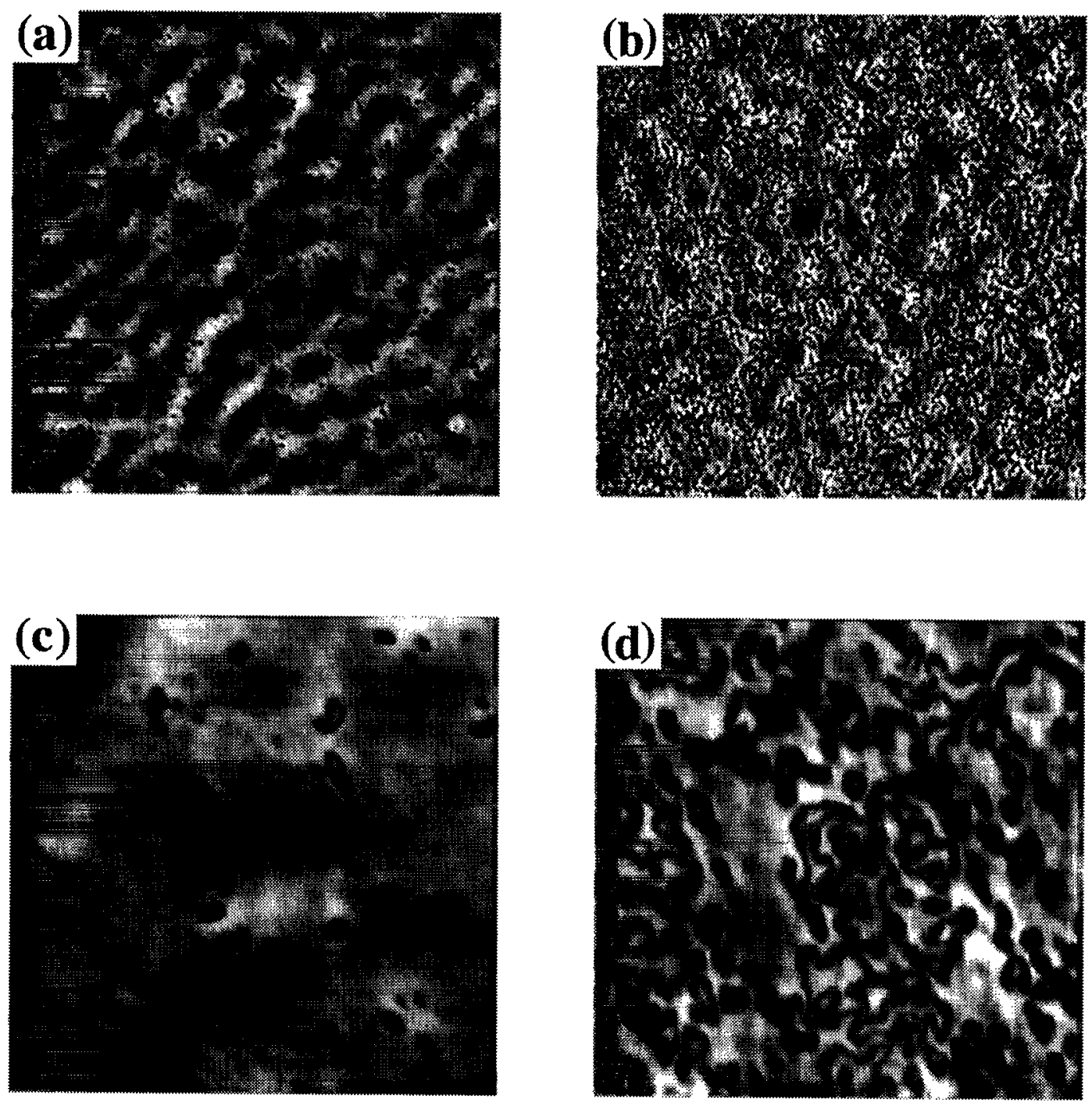

Fig. 1 


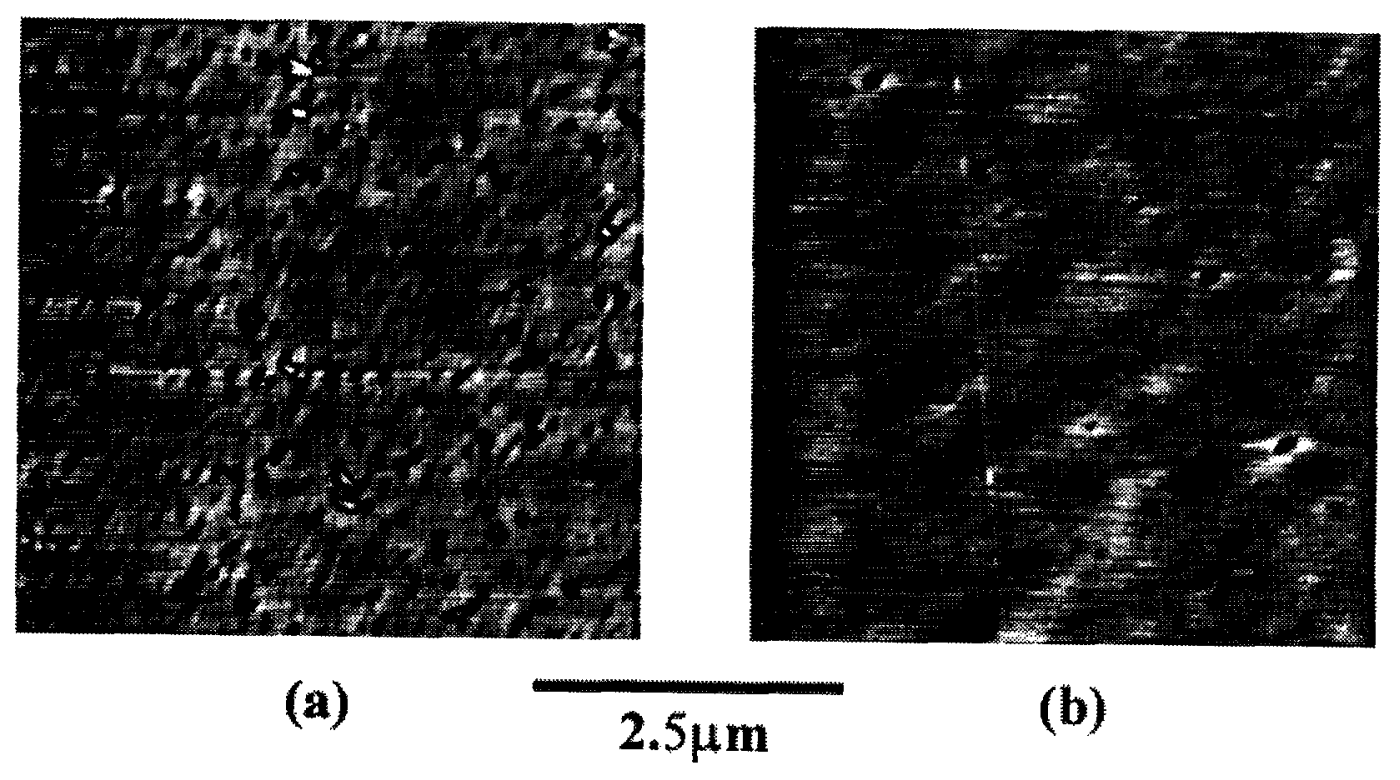

Fig. 2 


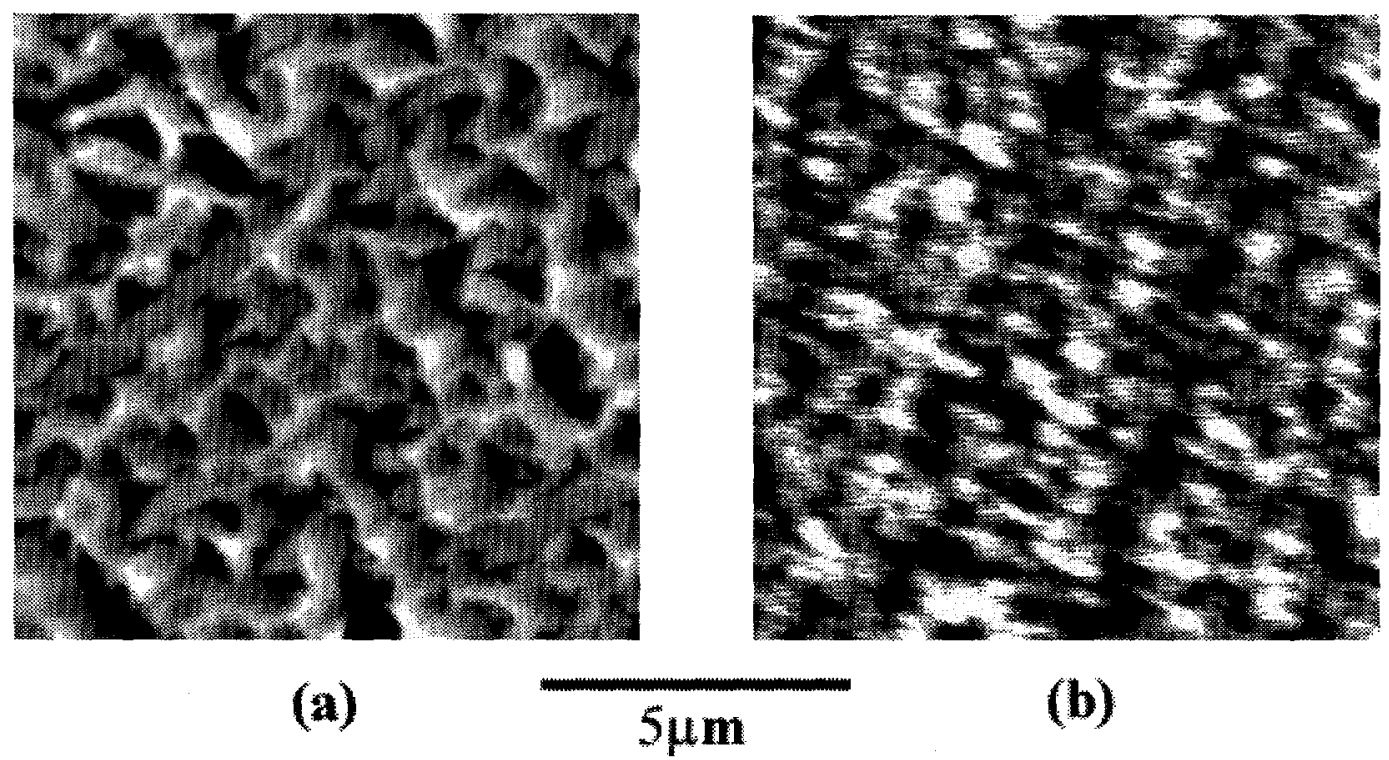

Fig. 3 

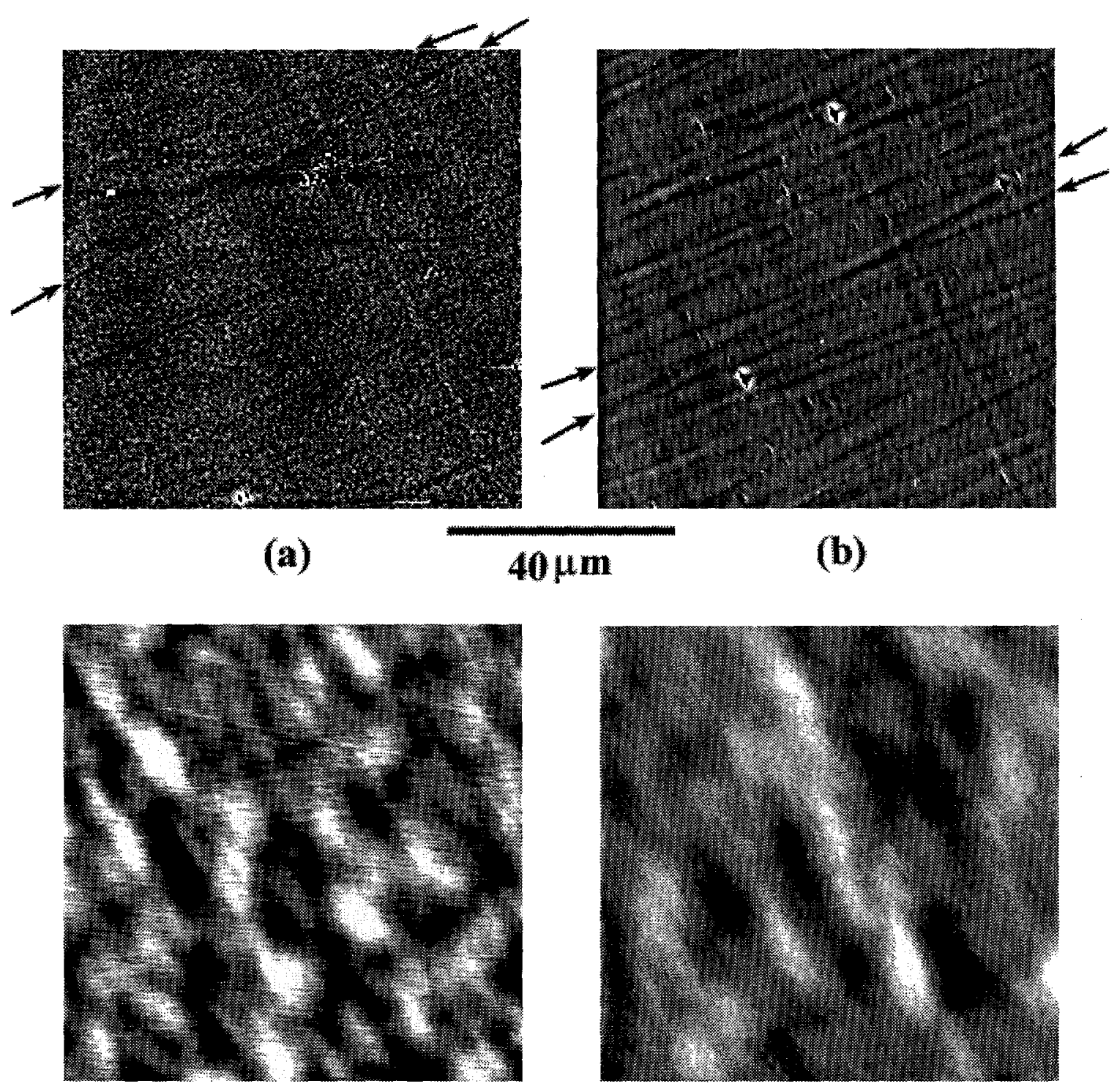

(c)

$$
2.5 \mu \mathrm{m}
$$

(d)

Fig. 4 